'Centro Universitário de Patos (Unifip) - Patos (PB), Brasil.

andredantas1.0@hotmail. com

2 Universidade Federal da Paraíba (UFPB) - Patos (PB), Brasil.

\section{Pagamento por desempenho às Equipes da Atenção Básica: análise a partir dos ciclos do PMAQ-AB}

\author{
Performance payment to the Primary Care Teams: analysis from the \\ $P M A Q-A B$ cycles
}

André Wagner Dantas Rodrigues ${ }^{\mathbf{1}}$ Emmanuella Costa de Azevedo Mello², Rozileide Martins

Simões Candeia², Geane Silva², Luciano Bezerra Gomes², Juliana Sampaio²

DOI: 10.1590/0103-1104202113109

\begin{abstract}
RESUMO O objetivo do estudo foi compreender como se deu o uso do recurso do Programa Nacional de Melhoria do Acesso e da Qualidade da Atenção Básica (PMAQ-AB) em nível municipal, como estratégia de pagamento por desempenho, no estado da Paraíba. Trata-se de um estudo descritivo, com abordagem quantitativa. Participaram do estudo 219 municípios, de um total de 223 existentes no estado, com os quais realizou-se um inquérito com os gestores municipais em 2018. Os dados sofreram análise estatística descritiva. Os resultados apontam que a maioria dos municípios adotou lei municipal como normativa para regulamentar o uso dos incentivos financeiros do PMAQ-AB. A avaliação externa foi o principal critério de repasse dos recursos financeiros às equipes de saúde. No decorrer dos ciclos, mais atores receberam recursos além da equipe mínima da estratégia de saúde da família e o repasse foi feito de forma semestral e anual, sendo cerca de $40 \%$ a $50 \%$ do valor destinado às equipes e o restante investido em melhorias das condições de trabalho. Conclui-se que os recursos do PMAQ-AB foram utilizados com uma tendência homogênea nos municípios da Paraíba, repassando incentivos financeiros para os trabalhadores, mas se preocupando também com investimentos nas condições de trabalho das equipes.
\end{abstract}

PALAVRAS-CHAVE Atenção Primária à Saúde. Remuneração. Gestão de recursos.

\begin{abstract}
The study aimed to understand how the resource of the National Program for Improving Access and Quality of Primary Care (PMAQ-AB) was used at the municipal level, as a payment-for-performance strategy, in the state of Paraiba. This is a descriptive study with a quantitative approach. 219 municipalities of a total of 223 in the state participated in the study, with which an inquiry was carried out with municipal managers in 2018. The data underwent descriptive statistical analysis. Results show that most municipalities have adopted a municipal law as a rule to regulate the use of financial incentives under the PMAQ-AB. External evaluation was the main criterion for transferring financial resources to health teams. During cycles, more actors received resources in addition to the minimum team of the family health strategy and the transfer was made on a biannual and annual basis, with around $40 \%$ to $50 \%$ of the amount allocated to the teams and the remainder invested in improvements in work conditions. It is concluded that the resources of the PMAQ-AB were used with a homogeneous trend in the municipalities of Paraiba, transferring financial incentives to workers, but also being concerned with investments in the working conditions of the teams.
\end{abstract}

KEYWORDS Primary Health Care. Remuneration. Resource management. 


\section{Introdução}

A saúde pública no Brasil, segundo Flôres et al. ${ }^{1}$ e Pinto, Sousa e Ferla ${ }^{2}$, tem sofrido dificuldades devido à insuficiência de recursos, às estruturas inadequadas das unidades de saúde e à deficiência no acesso à atenção. Dessa forma, no ano de 2011, com a intenção de aprimorar o Sistema Único de Saúde (SUS) e ampliar o acesso e a qualidade na Atenção Básica (AB), o então Governo Federal, administrado pela presidente Dilma Rousseff, criou o Programa Nacional de Melhoria do Acesso e da Qualidade da Atenção Básica (PMAQ-AB). O programa foi instituído pela Portaria ${ }^{\circ} 1.654$ GM/MS, de 19 de julho de 2011, e trouxe como principal objetivo:

Induzir a ampliação do acesso e melhoria da qualidade da atenção básica, com garantia de um padrão de qualidade comparável nacional, regional e localmente, de maneira a permitir maior transparência e efetividade das ações governamentais direcionadas à Atenção Básica à Saúde 3 .

O PMAQ-AB, logo quando lançado, estava estruturado em 4 fases, a saber: adesão e contratualização, desenvolvimento, avaliação externa e recontratualização, formando um ciclo que se completava. Essa estrutura se manteve durante os dois primeiros ciclos, porém no terceiro ciclo, o programa passou a ser constituído por 3 fases - adesão e contratualização, certificação e recontratualização sendo a fase de desenvolvimento transformada em um eixo transversal do programa ${ }^{\mathbf{3}, \mathbf{4}}$. Esta alteração se sustentou na tentativa de conferir maior destaque ao momento de desenvolvimento, considerado como estruturante do programa ${ }^{4}$, pois nele se esperava que ocorressem os processos de mudança da $\mathrm{AB}$.

O programa inovou, também, o processo de financiamento da $\mathrm{AB}$ ao adotar avaliações para repasse de recursos por meio de indicadores e metas estabelecidas, adicionando o componente de qualidade. As Equipes de Atenção Básica
(EAB) aderiram ao programa de maneira voluntária. Com a adesão das equipes, houve um aumento no valor repassado pelo Piso da Atenção Básica variado (PAB variado) para os municípios, de acordo com o desempenho alcançado ${ }^{5,2}$.

O PMAQ-AB utilizou como base para a transferência de recurso o pagamento por desempenho (Pay-For-Performance, P4P). Amstrong ${ }^{6}$ conceitua o pagamento por desempenho como um sistema de recompensa que atrela o repasse de recursos ao mérito de cada servidor ou equipe, aferido por meio de avaliações.

Poli-Neto et al. ${ }^{7}$, citam que o PMAQ-AB adotava uma forma de $\mathrm{P} 4 \mathrm{P}$ diferente do modelo tradicional, onde o recurso é transferido para os provedores ou serviço de saúde com base em metas ou ações determinadas. Nesse programa, o $\mathrm{P} 4 \mathrm{P}$ passou a ser uma forma de descentralização orçamentária em que os municípios decidiam sobre a aplicação dos incentivos financeiros variáveis alcançados. Essa forma de pagamento poderia estimular os trabalhadores e a gestão a alcançarem resultados cada vez mais significativos, podendo aumentar os valores de recursos recebidos de acordo com o desempenho das equipes. Contudo, Fontenele ${ }^{8}$ aponta que, em alguns estudos, os trabalhadores se mostram insatisfeitos com a distribuição dos recursos pela gestão, alegando que, ao invés do gestor se preocupar apenas com os acréscimos nos salários dos servidores, deveria incentivá-los de outras formas, como a partir de capacitação e melhoria das condições de trabalho.

O PMAQ-AB teve seu fim decretado no ano de 2019, após encerrar o seu $3^{\circ}$ ciclo de avaliação. O Ministério da Saúde, através da portaria $\mathrm{n}^{\mathrm{o}} 2.979$, de 12 de novembro de 2019, lançou o novo modelo de financiamento para a $\mathrm{AB}$, chamado Previne Brasil, que entrou em vigor a partir do ano $2020^{\circ}$, propondo utilizar como critério de repasse dos recursos a captação ponderada, o pagamento por performance, incentivo para ações estratégicas e provimento de profissionais de saúde ${ }^{10}$. 
Para o presente estudo, o estado da Paraíba foi escolhido por questão de conveniência dos pesquisadores. Além disso, a escolha se justifica pelas suas características, possuindo uma cobertura populacional de 94,0\% da Estratégia Saúde da Família (ESF) e por possuir um grande número de Núcleos de Apoio à Saúde da Família (Nasf) implantados: 152 Nasf-AB Modalidade I, 54 Nasf-AB Modalidade II e 78 Nasf-AB Modalidade III. Estes dados o tornam um estado representativo para uma pesquisa relacionada à $\mathrm{AB}$. Além disso, 77,2\% dos municípios do estado participaram do programa PMAQ-AB desde seu primeiro ciclo, evidenciando a ampla experiência deste no programa"1.

Como o PMAQ-AB não estabelece como os recursos repassados deverão ser aplicados pela gestão municipal, esta tem autonomia para investi-los de acordo com as necessidades locais. Diante desta flexibilização, o presente estudo objetiva compreender como se deu o uso do recurso do PMAQ-AB em nível municipal, como estratégia de pagamento por desempenho, no estado da Paraíba. Com isso, pretendemos ampliar a compreensão sobre as maneiras como esse programa pode ter afetado a $\mathrm{AB}$ no estado e contribuir com a literatura na identificação de possíveis modalidades mais frequentes de execução desses recursos, agregando na elaboração de modelos para avaliar o uso do PMAQ na gestão da AB no Brasil.

\section{Material e métodos}

O estudo é um recorte da pesquisa: 'Impactos do PMAQ-AB na Atenção Básica: análise da aplicação do incentivo financeiro por desempenho às equipes de saúde no Brasil'. Tratase de um estudo descritivo, com abordagem quantitativa. A base de dados da pesquisa foi construída pelos pesquisadores do Grupo de Pesquisa Política, Educação e Cuidado em Saúde (GPECS), da Universidade Federal da Paraíba (UFPB), a partir de questionário respondido por gestores municipais de saúde do estado da Paraíba.
O instrumento de coleta de dados é composto por quatro partes: a primeira parte contém questões que exploram o perfil do gestor municipal respondente; a segunda aborda a existência de normativas que regulamentam a gestão do recurso do PMAQ-AB no município; a terceira versa sobre a forma de aplicação dos recursos; e a quarta (e última) parte aborda a avaliação dos gestores dos efeitos causados pelos recursos na melhoria da $\mathrm{AB}$.

A coleta de dados foi realizada com cada participante entre os meses de julho e setembro de 2018, concomitante à avaliação externa do terceiro ciclo do PMAQ-AB na Paraíba. Para responder o questionário, o indivíduo deveria ser secretário de saúde ou alguém que representasse a gestão municipal nas questões relacionadas à política de AB. Cabe ressaltar, ainda, que a coleta de informações referentes aos ciclos 1,2 e 3 foi realizada de forma retrospectiva com os atuais gestores de 2018 .

A grande maioria dos respondentes $76,7 \%$ (168) - era do sexo feminino, com média de idade de 39,72 anos (Desvio Padrão $9,974)$ e variando entre 23 e 70 anos. Quanto ao cargo, 63,9\% (140) ocupavam a função de secretária(o) de saúde, 25,1\% (55) de direção/coordenação da AB, 5,9\% (13) de gerente e $5,0 \%$ (11) se encaixavam na opção 'outros cargos'. Para efeito deste estudo, esse conjunto de participantes será compreendido como os gestores municipais da $\mathrm{AB}$.

Para coletar as informações, a equipe de avaliação externa solicitava que os gestores respondessem a um questionário aplicado pelo próprio avaliador. Quando o gestor afirmava não ter condições de respondê-lo naquele momento, era fornecido um link de acesso ao formulário, via WhatsApp, para que fosse respondido posteriormente.

Os dados coletados foram organizados e agrupados através do programa Excel, na versão 2016. Para a análise de dados, utilizou-se a técnica estatística de análise descritiva, recorrendo aos cálculos de 
médias e valores relativos (percentuais e desvios padrões) das variáveis em estudo. Essa estratégia foi proposta segundo o seu potencial explicativo, permitindo a visualização, de forma mais clara e objetiva, dos desfechos do estudo e suas interpretações ${ }^{\mathbf{1 2}}$.

Ressalta-se que a pesquisa originária do banco cumpriu os aspectos éticos da Resolução ${ }^{0}$ 466/12 do Conselho Nacional de Saúde, sendo submetida ao Comitê de Ética em Pesquisa do Centro de Ciências da Saúde - CEP/CCM/UFPB e aprovada com registro de Certificado de Apresentação para Apreciação Ética de número 90331418.6.0000.8069.

Ressalta-se, ainda, que esta pesquisa não obteve financiamento específico de agências de fomento para seu desenvolvimento, porém parte do seu trabalho de campo foi realizado em paralelo às ações de avaliação externa do terceiro ciclo do PMAQ-AB, os quais, foram realizados com o financiamento do Ministério da Saúde.

\section{Resultados e discussão}

Os resultados desse estudo foram agregados em temas e serão apresentados em quatro partes, para uma melhor abordagem e discussão dos dados: (a) modos de aplicação dos recursos operados pelos municípios da Paraíba; (b) tipos de legislações utilizadas para o repasse dos recursos no município; (c) critérios de avaliação e de repasse dos recursos do PMAQ-AB; (d) avaliação dos gestores sobre os efeitos do PMAQ-AB na qualificação da AB.

\section{Modos de aplicação dos recursos operados pelos municípios da Paraíba}

Dado que o PMAQ-AB não determinava a forma de aplicação dos recursos financeiros, cabendo ao gestor municipal essa tomada de decisão, é importante compreender como foi realizada a definição do repasse do incentivo para as EAB. Os dados da tabela 1 demonstram como isso se deu na Paraíba.

Tabela 1. Resultado da definição do repasse concebido pelo PMAQ-AB para as Equipes de Atenção Básica

\begin{tabular}{lrrrrrr}
\hline & \multicolumn{3}{c}{ Ciclos do PMAQ-AB } \\
Como é definido o repasse do incentivo financeiro do & Ciclo 1 & \multicolumn{3}{c}{ Ciclo 2 } & \multicolumn{2}{c}{ Ciclo 3 } \\
PMAQ-AB? & $\mathbf{n}$ & $\mathbf{6}$ & $\mathbf{n}$ & $\mathbf{\%}$ & $\mathbf{n}$ & $\mathbf{\%}$ \\
\hline Valor fixo para todos os profissionais & 50 & 22,8 & 58 & 26,5 & 53 & 24,2 \\
Percentual sobre a remuneração mensal individual de & 46 & 21,0 & 67 & 30,6 & 68 & 31,1 \\
cada profissional & & & & & & \\
Não há repasse para as EAB & 27 & 12,3 & 02 & 0,9 & 10 & 4,6 \\
Outros & 39 & 17,8 & 57 & 26,0 & 61 & 27,9 \\
NS/NR & 57 & 26,0 & 35 & 16,0 & 27 & 12,3 \\
Total & 219 & 100 & 219 & 100 & 219 & 100 \\
\hline
\end{tabular}

Fonte: Elaboração própria.

Os resultados mostram que, nos três ciclos, as formas mais frequentes de repasse dos incentivos financeiros aos trabalhadores eram por um valor fixo ou por um percentual sobre a remuneração mensal. Observa-se, ainda, que os valores entre essas duas opções de respostas encontram-se bem próximos e vão se distanciando com o passar dos ciclos, com destaque para a opção percentual sobre a remuneração mensal, que aumentou sua diferença de $1,8 \%$ no primeiro ciclo para $6,9 \%$ no terceiro ciclo. Isso mostra que houve um aumento na 
diferença entre os valores repassados para os diferentes trabalhadores das equipes.

Esse critério de repasse pode gerar insatisfação nos trabalhadores, pois o fato de se esforçarem em conjunto, mas receberem valores diferentes (percentual sobre remuneração mensal) produz tensões internas nas equipes, gerando disputas e competições por bônus individuais, que podem prejudicar o trabalho em equipe ${ }^{13,14}$. Destaca-se que o trabalho individual não era capaz de promover as mudanças de interesse do PMAQ-AB, cabendo, portanto, a toda a equipe o envolvimento no processo que repercutisse na organização do serviço como um todo ${ }^{15}$.

Os resultados que se referem às $\mathrm{EAB}$ que receberam os recursos do $\mathrm{PMAQ-AB}$ evidenciam que grande parte dos gestores municipais repassaram os recursos de forma exclusiva para as equipes participantes do programa. Observou-se que, no $1^{\circ}$ ciclo, 53,9\% (118) dos gestores repassavam recursos para as EAB e esse critério sofreu um aumento ao longo dos ciclos. No $2^{\circ}$ ciclo, a taxa foi de $71,7 \%$ (157) e no $3^{\circ}$ ciclo $73,1 \%$ (160), assim como o valor de resposta para a alternativa 'não houve repasse para as equipes' foi diminuindo com a transição dos ciclos.

Esses dados evidenciam que o pagamento por desempenho, através de repasse de recursos para os trabalhadores, passou a ser adotado cada vez mais pelos municípios paraibanos. A partir disso, geram-se reflexões sobre o real motivo da adoção do pagamento por desempenho por parte dos gestores, haja vista a pressão dos servidores para receber recursos, municípios vizinhos adotando essa forma de pagamento, ou mesmo uma compreensão dos gestores da sobre os benefícios de um programa de $\mathrm{P} 4 \mathrm{P}$.
Armond ${ }^{16}$ e Bertusso \& Rizzotto ${ }^{17}$ apontam que são vários os motivos para a adoção do pagamento por desempenho no setor saúde, pois sua contribuição vai desde motivar os trabalhadores aumentando seu empenho nos serviços, por meio de um aumento ou atribuição de remuneração, até a melhoria na qualidade dos serviços oferecidos pela instituição como um todo. Contudo, Barreto ${ }^{18}$, Zermiani, Baldani e Ditterich ${ }^{19}$ ressaltam que o pagamento por desempenho requer um planejamento dos incentivos que serão repassados aos trabalhadores, sob pena de gerar efeitos inesperados, como desmotivar o trabalhador a realizar tarefas que são importantes, porém não remuneradas, fraude na apresentação de relatórios, seleção de pacientes para a facilidade de atingir objetivos e até mesmo causar uma dependência dos recursos financeiros.

No contexto da falsificação na apresentação dos dados, percebe-se que alguns autores relatam a existência de uma manipulação da realidade local, como é o caso da 'PMAQuiagem', citada por Sampaio et al. ${ }^{20}$. Essa 'PMAQuiagem' forjava a percepção dos avaliadores, produzindo resultados mais favoráveis na avaliação externa. Assim, o município não deixaria de receber os recursos anteriores e poderia ampliá-los, o que mostra também uma dependência de recursos das equipes.

Neves, Montenegro e Bittencourt 21,22 ressaltam que o modelo de gestão baseado no cumprimento de objetivos, visando apenas o quantitativo de procedimentos, sem reuniões frequentes entre os atores envolvidos para gerar reflexões sobre a melhoria do processo de trabalho, pode gerar problemas entre os gestores e os profissionais de saúde.

As informações expostas a seguir apontam o percentual dos incentivos que são repassados para as EAB (tabela 2). 
Tabela 2. Percentual dos incentivos financeiros do PMAQ-AB repassado para as Equipes de Atenção Básica

\begin{tabular}{|c|c|c|c|c|c|c|}
\hline \multirow[b]{3}{*}{ Percentual repassado } & \multicolumn{6}{|c|}{ Ciclos do PMAQ-AB } \\
\hline & \multicolumn{2}{|c|}{ Ciclo 1} & \multicolumn{2}{|c|}{ Ciclo 2} & \multicolumn{2}{|c|}{ Ciclo 3} \\
\hline & $\mathrm{n}$ & $\%$ & $\mathrm{n}$ & $\%$ & $\mathbf{n}$ & $\%$ \\
\hline De 0 a 20\% & 05 & 2,3 & 06 & 2,7 & 04 & 1,8 \\
\hline De 20 a $30 \%$ & 15 & 6,8 & 18 & 8,2 & 18 & 8,2 \\
\hline De 30 a $40 \%$ & 13 & 5,9 & 21 & 9,6 & 22 & 10,0 \\
\hline De 40 a $50 \%$ & 59 & 26,9 & 80 & 36,5 & 84 & 38,4 \\
\hline De 50 a 60\% & 21 & 9,6 & 32 & 14,6 & 31 & 14,2 \\
\hline De 60 a $70 \%$ & 06 & 2,7 & 12 & 5,5 & 10 & 4,6 \\
\hline De 70 a $100 \%$ & 07 & 3,2 & 08 & 3,7 & 07 & 3,2 \\
\hline Não houve repasse paras as equipes & 25 & 11,4 & 02 & 0,9 & 08 & 3,7 \\
\hline NS/NR & 68 & 31,1 & 40 & 18,3 & 35 & 16,0 \\
\hline
\end{tabular}

Fonte: Elaboração própria.

Analisando de modo específico as alternativas de respostas fornecidas, verifica-se que a maioria dos gestores municipais designaram um percentual de $40 \%$ a $50 \%$ do valor recebido do $\mathrm{PMAQ}-\mathrm{AB}$ para as $\mathrm{EAB}$ (tabela 2). É possível compreender que houve avanço gradual de adesão dos municípios nesta modalidade de percentual de repasse, conforme a ocorrência dos ciclos.

A decisão de destinar uma parcela do incentivo para o pagamento dos profissionais de saúde tem sido amplamente discutida pela literatura científica. Diversos estudos apontam que o recurso financeiro repassado sobre um percentual variável é pactuado por vários municípios, como forma de um mecanismo para incentivar a adesão dos trabalhadores das $\mathrm{EAB}$ ao processo ${ }^{2,17,18}$.

Esses incentivos poderiam ser repassados para os trabalhadores das EAB ou serem utilizados em outros investimentos na $\mathrm{AB}$, por exemplo, aplicando-os como fomento contínuo à qualificação da assistência ofertada neste âmbito. Entretanto, essa parcela de recursos, quando destinada às equipes como forma de pagamento por desempenho, é capaz de incentivar a alcançar os objetivos estabelecidos ${ }^{21}$.

Nesse contexto, percebe-se que os outros $50 \%$ a $60 \%$ dos recursos tendem a ser investidos nas condições de trabalho e na educação permanente em saúde. Essa distribuição do restante do recurso é de suma importância, pois viabiliza a resolução de problemas de menor complexidade dos pacientes e pode atingir os objetivos pactuados e, consequentemente, o sucesso de uma atividade, programa ou projeto na $\mathrm{AB}$ de saúde $21,23,24$.

Os resultados apontaram para uma homogeneidade em três opções de respostas (reparos nas Unidades Básicas de Saúde - UBS, processos de educação permanente em saúde e compras de medicamentos, insumos e materiais) nos três ciclos de avaliação. Desta forma, percebe-se que, possivelmente, na Paraíba, existia um padrão para o investimento do restante dos recursos na melhoria dos processos de trabalho conforme a decorrência dos ciclos.

Essa forma de utilização do restante dos recursos se mostra positiva para a melhoria das condições de trabalho das equipes participantes do programa. Neste âmbito, destaca-se que é essencial para qualquer trabalhador ter uma boa estrutura e oferta de materiais para a entrega de qualidade nos serviços. Estes, inclusive, são pontos bastante discutidos na gestão do trabalho da $\mathrm{AB}$, como citado no trabalho de Frigoto ${ }^{25}$.

Vale ressaltar que as condições de trabalho na Atenção Básica de Saúde (ABS) são 
aspectos bastante comprometidos desde o surgimento do SUS, devido à ocorrência do subfinanciamento na saúde. A questão da limitação dos recursos acaba acarretando vários problemas na oferta de serviços à população e, nos últimos anos, após a aprovação da Emenda Constitucional 95 (EC 95) isso parece ainda pior, com a imediata diminuição na cobertura de vacinas e o aumento na mortalidade infantil26,27.

É de suma importância destacar, também, o investimento na educação permanente. Esta tem a finalidade de produzir mudanças no setor da saúde, ampliando a capacidade de autoavaliação e autogestão dos trabalhadores, capacitando os profissionais e qualificando o processo de trabalho da equipe. Além disso, ela é instituída com uma estratégia importante na estimulação dos envolvidos no processo, causando uma reflexão crítica sobre o que precisa ser transformado e melhorado ${ }^{\mathbf{2 8 , 2 9}}$.

Jales, Jales e Gomes ${ }^{15}$ ressaltam que o PMAQ-AB não considerava a educação permanente apenas como participação em cursos, mas como parte de uma estratégia de gestão através de planejamento e programação de trabalhos coletivos para resolução de problemas e necessidades de saúde sofridas pela população.

Na tabela 3, abaixo, estão descritos os resultados referentes à distribuição do recurso entre os profissionais da $\mathrm{AB}$.

Tabela 3. Distribuição do incentivo do PMAQ-AB entre os profissionais que compõem as equipes de saúde, de acordo com os ciclos de avaliação

\begin{tabular}{|c|c|c|c|c|c|c|}
\hline \multirow[b]{3}{*}{ Profissionais da saúde } & \multicolumn{6}{|c|}{ Ciclos do PMAQ-AB } \\
\hline & \multicolumn{2}{|c|}{ Ciclo 1} & \multicolumn{2}{|c|}{ Ciclo 2} & \multicolumn{2}{|c|}{ Ciclo 3} \\
\hline & $\mathbf{n}$ & $\%$ & $\mathbf{n}$ & $\%$ & $\mathbf{n}$ & $\%$ \\
\hline Médico & 124 & 13,1 & 164 & 12,6 & 158 & 11,8 \\
\hline Enfermeiro & 137 & 14,4 & 179 & 13,7 & 176 & 13,1 \\
\hline Odontólogos & 135 & 14,2 & 179 & 13,7 & 179 & 13,3 \\
\hline Técnico em Enfermagem & 70 & 7,4 & 170 & 13,0 & 170 & 12,7 \\
\hline Agente Comunitário de Saúde & 125 & 13,2 & 167 & 12,8 & 170 & 12,7 \\
\hline Técnico em Saúde Bucal & 124 & 13,1 & 168 & 12,9 & 169 & 12,6 \\
\hline Equipe Nasf & 57 & 6,0 & 95 & 7,3 & 125 & 9,3 \\
\hline Outros Profissionais de Nível Superior & 28 & 2,9 & 41 & 3,1 & 49 & 3,7 \\
\hline Gerente & 62 & 6,5 & 86 & 6,6 & 84 & 6,3 \\
\hline Não há repasse financeiro & 29 & 3,1 & 01 & 0,1 & 13 & 1,0 \\
\hline Outros profissionais & 31 & 3,3 & 47 & 3,4 & 48 & 3,6 \\
\hline NS/NR & 28 & 2,9 & 10 & 0,8 & 0 & 0 \\
\hline
\end{tabular}

Fonte: Elaboração própria.

As informações contidas na tabela 3 apontam que, ao longo dos ciclos, os repasses do incentivo financeiro do PMAQ-AB foram destinados a mais atores, além da equipe mínima (médicos, enfermeiros, técnicos e equipe de saúde bucal). Este dado pode estar relacionado a questões relatadas anteriormente, como a pressão das categorias profissionais para receber recursos, a adoção do $\mathrm{P} 4 \mathrm{P}$ como incentivo à implantação de boas práticas em saúde, ou mesmo a valorização, por parte dos gestores, do incentivo ao trabalho em equipe envolvendo outros trabalhadores no esquema de recompensa do $\mathrm{P} 4 \mathrm{P}$.

Os dados expressos na questão 'Para quem ou para qual outro serviço foram repassados 
incentivos financeiros além da $\mathrm{EAB}$ ?' apontam que poucos municípios tendiam a repassar recursos para outros serviços além das EAB. Porém, nota-se que os municípios que faziam esse repasse para além das EAB acabavam investindo de alguma forma na $\mathrm{AB}$. Entre os agentes que recebiam recursos, além das $\mathrm{EAB}$, estão: no $1^{\circ}$ ciclo, a 'equipe de apoio institucional', com 2,7\% (06) dos respondentes; no $2^{\circ}$ ciclo, a 'equipe de gestores da $\mathrm{AB}$ ', com $4,1 \%$ (09) dos respondentes; e no $3^{\circ}$ ciclo, 'Agentes de Combate a Endemias (ACE), Núcleo de
Apoio à Saúde da Família (Nasf) e Centro Especializado de Odontologia (CEO)', com $5,5 \%$ (12) dos respondentes. Sobre os resultados, sugere-se estudos que avaliem se tal divisão foi capaz de proporcionar benefícios ou conflitos em termos de processos de trabalho, haja vista a realidade local de cada município.

A seguir, na tabela 4, estão expostos os resultados referentes à periodicidade com que esses recursos eram repassados pelo município para as equipes.

Tabela 4. Periodicidade repasse do incentivo financeiro do PMAQ-AB às equipes

\begin{tabular}{|c|c|c|c|c|c|c|}
\hline \multirow[b]{3}{*}{ Periodicidade } & \multicolumn{6}{|c|}{ Ciclos do PMAQ-AB } \\
\hline & \multicolumn{2}{|c|}{ Ciclo 1} & \multicolumn{2}{|c|}{ Ciclo 2} & \multicolumn{2}{|c|}{ Ciclo 3} \\
\hline & n & $\%$ & $\mathbf{n}$ & $\%$ & n & $\%$ \\
\hline Mensal & 30 & 15,3 & 36 & 18,3 & 27 & 13,7 \\
\hline Bimestral & 09 & 4,6 & 09 & 4,6 & 09 & 4,6 \\
\hline Trimestral & 16 & 8,2 & 19 & 9,6 & 20 & 10,2 \\
\hline Semestral & 42 & 21,4 & 65 & 33,0 & 72 & 36,5 \\
\hline Anualmente & 34 & 17,3 & 51 & 25,9 & 49 & 24,9 \\
\hline Vinculada à avaliação externa do PMAQ-AB & 03 & 1,5 & 01 & 0,5 & 01 & 0,5 \\
\hline Não há periodicidade definida & 26 & 13,3 & 02 & 1,0 & 03 & 1,5 \\
\hline Não há repasse para as equipes & 36 & 18,4 & 03 & 1,5 & 11 & 5,6 \\
\hline
\end{tabular}

Fonte: Elaboração própria.

Os resultados (tabela 4) apontam que o repasse semestral era o mais utilizado nos três ciclos. Entretanto, percebe-se que o pagamento anual possui valores de respostas bem próximos da opção semestral. Além disso, é possível observar que, nos dois casos, houve um aumento paulatino com o decorrer dos ciclos de avaliação. Os dados ainda revelam que, no $1^{\circ}$ ciclo, $13,3 \%$ (26) dos gestores municipais não tinham periodicidade definida de repasse e que este comportamento foi reduzido na ocorrência do $2^{\circ}-\operatorname{com} 1,0 \%$ (02) - e do $3^{\circ}$ ciclo - este com 1,5\% (03). Esse fato pode estar relacionado à criação/definição de normativas por cada município, uma vez que passou a haver um padrão de distribuição.

Não foram encontrados estudos que ajudassem a compreender a opção por essa periodicidade do pagamento. Contudo, estima-se que a experiência dos gestores ao longo dos ciclos do PMAQ-AB, aliada às discussões oportunizadas entre os atores participantes e ao conhecimento adquirido neste caminho, foram conformando esta definição da periodicidade dos repasses financeiros. Esta reflexão apoia-se no fato de que os dados mostram uma variação positiva dos percentuais de respostas dos gestores municipais, conforme a ocorrência dos ciclos para todos os municípios participantes da amostra.

\section{Legislação}

No quesito relacionado à existência de normativas no município que regulamentem o 
uso dos incentivos financeiros do PMAQ-AB, $47,0 \%$ (103) apontam a existência dessas normativas desde o primeiro ciclo de avaliação, chegando a 79,0\% (173) no segundo ciclo e 86,3\% (189) no terceiro ciclo. Durante a análise das as normativas existentes, percebeu-se que a lei municipal foi a mais utilizada nos três ciclos $-1^{\circ}$ ciclo com $41,6 \%$ (91), $2^{\circ}$ ciclo com $72,6 \%$ (159) e $3^{\circ}$ ciclo com $74,0 \%$ (162) -, seguida da pactuação do conselho municipal de saúde $-1^{\circ}$ ciclo com $1,8 \%$ (04), $2^{\circ}$ ciclo com $2,7 \%$ (06) e $3^{\circ}$ ciclo com $5,5 \%$ (12).

Moraes e Iguti ${ }^{30}$ ressaltam que o PMAQ-AB não pressupunha que os incentivos financeiros recebidos pelos municípios fossem repassados para suas equipes de saúde, mesmo sendo estes recursos alcançados a partir da avaliação do desempenho delas ${ }^{17}$. Assim, o sistema introduzido pelo PMAQ-AB demandava dos gestores municipais de saúde a adequação ao novo e a criação de legislações próprias, baseadas na realidade e nas necessidades locais. Nesse sentido, cada município definia os percentuais e áreas nas quais os recursos deveriam ser aplicados, norteando os possíveis investimentos e o repasse de gratificações aos trabalhadores ${ }^{17}$.

Para Reis ${ }^{31}$, o fato de o município adotar uma normativa regulamentando o repasse dos recursos financeiros concedidos pelo programa para as equipes, apresentava-se como um ponto positivo. Isto porque a existência de uma norma tenderia a evitar maiores descontentamentos dos profissionais e desconforto no processo de trabalho, melhorando o diálogo entre gestão e trabalhadores, permitindo a estes últimos saberem como se dava a destinação dos recursos.

\section{Critérios de avaliação e de repasse dos recursos do PMAQ-AB}

Dentre os respondentes, grande maioria, correspondente a $76,7 \%$ (168), respondeu que não havia no município incentivo financeiro adicional para os trabalhadores antes do PMAQAB. Dos que afirmaram que havia algum tipo de incentivo antes do programa, a maior parte foi composta por gestores de municípios de pequeno e médio porte (até 25 mil habitantes e de 25 mil a 100 mil habitantes, respectivamente), tendo apenas João Pessoa e Campina Grande com características de grande porte (acima de 100 mil habitantes). Ainda, dentre os que afirmaram que havia incentivo financeiro antes do PMAQ-AB, 67,5\% (29) responderam que o recurso era repassado para os trabalhadores em forma de bônus, prêmio e gratificação; os demais 32,5\% (14) Não Sabiam ou Não Responderam (NS/NR). Esses dados mostram que a maioria dos municípios não utilizava o pagamento por performance e o PMAQ-AB surge com uma proposta positiva para atribuir incentivos financeiros aos trabalhadores.

Machado $^{32}$ aponta que o fato de atribuir bônus, prêmio ou gratificação motiva as $\mathrm{EAB}$ a designarem sistemas de verificação do processo de trabalho e atenção à saúde da população de sua área de abrangência, de acompanhamento e avaliação de indicadores, de participação da comunidade e de satisfação do usuário.

Pinto, Magalhães e Koerner ${ }^{33}$ pontuam que o PMAQ-AB ocasionou um aumento nos recursos de cada equipe que aderisse ao programa. Além disso, os autores complementam que o programa foi capaz de causar um impacto positivo no financiamento geral da $\mathrm{AB}$. Esse impacto positivo pode ser observado através da comparação entre anos de 2010 e 2014, onde, no ano de 2011, o repasse foi de 70 milhões de reais, em 2012, chegou a quase 700 milhões e, em 2014, a estimativa foi de 1,6 bilhão. Para esse período, foi estimado um aumento do $\mathrm{PAB}$ variado de 5,92 para 11,17 bilhões de reais. Os autores complementam que $30 \%$ desse aumento foi ocasionado pela criação do componente de qualidade do programa.

Segundo os gestores municipais, a avaliação externa do PMAQ-AB foi o principal critério utilizado para a definição do repasse financeiro do programa para as EAB, sendo paulatinamente o mais utilizado no decorrer dos ciclos: $47,0 \%$ (103) no $1^{\circ}$ ciclo, $57,5 \%$ (126) no $2^{\circ}$ ciclo e $56,6 \%$ (124) no $3^{\circ}$ ciclo. Outro critério utilizado 
foi o alcance de metas absolutas e/ou relativas: $8,7 \%$ (19) no $1^{\circ}$ ciclo, $15,1 \%$ (33) no $2^{\circ}$ ciclo e $16,0 \%$ (35) no $3^{\circ}$ ciclo. Nesse sentido, a opção de distribuir o recurso igualmente a todas as $\mathrm{EAB}$ foi a menos indicada pelos respondentes: $7,3 \%$ (16) no $1^{\circ}$ ciclo, $11,0 \%$ (24) no $2^{\circ}$ ciclo e $10,0 \%$ (22) no $3^{\circ}$ ciclo. O conjunto desses dados evidencia que a medida da performance das equipes (seja pelo resultado da avaliação externa ou pelo alcance de metas absolutas e/ ou relativas) foi um importante critério para o repasse de recursos, o que caracteriza o pagamento por desempenho.

Alguns autores apontam que, com o passar dos anos, estratégias como o pagamento por desempenho e a contratualização de resultados têm sido adotadas na $A B$, na busca por uma maior qualidade dos serviços ofertados à população ${ }^{\mathbf{3 1} 33}$. Nesta perspectiva, o PMAQ-AB, ao adotar a lógica de recompensa do $\mathrm{P} 4 \mathrm{P}$ a partir da avaliação de desempenho das equipes de saúde, parecia buscar incentivar a melhoria do acesso e da qualidade na $\mathrm{AB}$ a partir do fortalecimento das práticas de monitoramento e avaliação e o cumprimento de metas estabelecidas $20,29,34,35$.

\section{Efeitos do PMAQ-AB na qualificação da $A B$}

Em seu último bloco de perguntas, o instrumento desse estudo abordou a percepção dos gestores sobre se os recursos do PMAQ-AB incentivaram melhorias na $\mathrm{AB}$, conforme a tabela 5 , a seguir.

Tabela 5. Avaliação do gestor quanto ao incentivo da qualificação das equipes a partir do recurso financeiro do PMAQ-AB

\begin{tabular}{|c|c|c|c|c|c|c|}
\hline \multirow[b]{3}{*}{ Variáveis } & \multicolumn{6}{|c|}{ Ciclos do PMAQ-AB } \\
\hline & \multicolumn{2}{|c|}{ Ciclo 1} & \multicolumn{2}{|c|}{ Ciclo 2} & \multicolumn{2}{|c|}{ Ciclo 3} \\
\hline & $\mathrm{n}$ & $\%$ & $\mathrm{n}$ & $\%$ & $\mathrm{n}$ & $\%$ \\
\hline \multicolumn{7}{|l|}{ Melhoria do Acesso } \\
\hline Não incentiva nenhuma melhora & 52 & 23,7 & 48 & 21,9 & 33 & 15,1 \\
\hline Indiferente & 30 & 13,7 & 39 & 17,8 & 42 & 19,2 \\
\hline Incentiva a melhora & 77 & 35,2 & 112 & 51,1 & 127 & 58,0 \\
\hline NS/NR & 60 & 27,4 & 20 & 9,1 & 17 & 7,8 \\
\hline \multicolumn{7}{|l|}{ Melhoria da Eficiência } \\
\hline Não incentiva nenhuma melhora & 48 & 21,9 & 49 & 22,4 & 34 & 15,5 \\
\hline Indiferente & 33 & 15,1 & 37 & 16,9 & 39 & 17,8 \\
\hline Incentiva a melhora & 78 & 35,6 & 114 & 52,1 & 129 & 58,9 \\
\hline NS/NR & 60 & 27,4 & 19 & 8,7 & 17 & 7,8 \\
\hline \multicolumn{7}{|l|}{ Melhoria dos processos de Trabalho } \\
\hline Não incentiva nenhuma melhora & 47 & 21,5 & 48 & 21,9 & 31 & 14,2 \\
\hline Indiferente & 30 & 13,7 & 35 & 16,0 & 39 & 17,8 \\
\hline Incentiva a melhora & 83 & 37,9 & 116 & 53,0 & 133 & 60,7 \\
\hline NS/NR & 59 & 26,9 & 20 & 9,1 & 16 & 7,3 \\
\hline \multicolumn{7}{|l|}{ Melhoria dos Resultados em Saúde } \\
\hline Não incentiva nenhuma melhora & 46 & 21,0 & 50 & 22,8 & 36 & 16,4 \\
\hline Indiferente & 29 & 13,2 & 30 & 13,7 & 33 & 15,1 \\
\hline Incentiva a melhora & 85 & 38,8 & 121 & 55,3 & 134 & 61,2 \\
\hline NS/NR & 59 & 26,9 & 18 & 8,2 & 16 & 7,3 \\
\hline
\end{tabular}


Tabela 5. (cont.)

\begin{tabular}{|c|c|c|c|c|c|c|}
\hline \multirow[b]{3}{*}{ Variáveis } & \multicolumn{6}{|c|}{ Ciclos do PMAQ-AB } \\
\hline & \multicolumn{2}{|c|}{ Ciclo 1} & \multicolumn{2}{|c|}{ Ciclo 2} & \multicolumn{2}{|c|}{ Ciclo 3} \\
\hline & n & $\%$ & $\mathbf{n}$ & $\%$ & $\mathbf{n}$ & $\%$ \\
\hline \multicolumn{7}{|l|}{ Melhoria da Satisfação do Usuário } \\
\hline Não incentiva nenhuma melhora & 46 & 21,0 & 49 & 22,4 & 32 & 14,6 \\
\hline Indiferente & 32 & 14,6 & 33 & 15,1 & 41 & 18,7 \\
\hline Incentiva a melhora & 81 & 37,0 & 117 & 53,4 & 129 & 58,9 \\
\hline NS/NR & 60 & 27,4 & 20 & 9,1 & 17 & 7,8 \\
\hline \multicolumn{7}{|l|}{ Melhoria da Satisfação Profissional } \\
\hline Não incentiva nenhuma melhora & 48 & 21,9 & 47 & 21,5 & 30 & 13,7 \\
\hline Indiferente & 27 & 12,3 & 31 & 14,2 & 37 & 16,9 \\
\hline Incentiva a melhora & 84 & 38,4 & 120 & 54,8 & 136 & 62,1 \\
\hline NS/NR & 60 & 27,4 & 21 & 9,6 & 16 & 7,3 \\
\hline
\end{tabular}

Fonte: Elaboração própria.

Os resultados mostram que os gestores afirmaram que o PMAQ-AB incentivou a melhoria em todas as categorias questionadas. Nos dois primeiros ciclos, percebe-se que a categoria que obteve maiores valores de resposta foi 'melhoria nos resultados de saúde', com 38,8\% (85) e $55,3 \%$ (121) dos respondentes, respectivamente. Já no terceiro ciclo, percebe-se que o maior valor de respostas foi atribuído à 'melhoria da satisfação profissional', com 62,1\% (136) dos respondentes.

Percebe-se, ainda, que os valores para as opções de resposta 'não incentiva nenhuma melhora' e 'NS/NR', em todos os aspectos questionados, são bem semelhantes nos dois primeiros ciclos e vão diminuindo com o passar dos ciclos do programa.

O trabalho de Pinto, Sousa e Ferla ${ }^{2}$ apresenta alguns dados de resposta de gestores municipais, em âmbito nacional, que se referem à melhoria ocasionada pela implementação do PMAQ-AB. Os avaliados classificaram alguns pontos, tais quais: a redução no tempo de espera nas UBS, a ampliação do número de pessoas atendidas pelas EAB e a melhora no acesso e na humanização do atendimento aos usuários. Esses pontos foram conceituados pelos respondentes em muita ou moderada melhoria, corroborando, assim, os achados deste estudo.
Percebe-se com os dados levantados que, na visão dos gestores municipais, o programa conseguiu atingir os objetivos específicos traçados, dentre os quais fornecer padrões de boas práticas e organização das UBS, ampliar o impacto da $\mathrm{AB}$ sobre as condições de saúde da população e também na satisfação de seus usuários além de estimular o foco da procura da $\mathrm{AB}$ pelo usuário ${ }^{3}$.

Cabe ressaltar que o PMAQ-AB foi além de um programa de pagamento por desempenho, sendo uma inovação na gestão da $\mathrm{AB}$, que buscou fomentar diferentes processos: institucionalização do monitoramento e avaliação, Avaliação da Melhoria da Qualidade (AMQ), ações de educação permanente e apoio institucional, além do pagamento por desempenho, com o intuito de mobilizar os indivíduos envolvidos em uma dinâmica de gestão, atuação e negociação que impulsionasse de forma permanente a mudança ${ }^{36}$.

Como limitações do estudo, indica-se: 1.0 fato de se tratar de uma coleta de dados transversal sobre aspectos não apenas do momento, mas também referentes a anos anteriores, podendo incidir em viés de memória, o que ocasionou um número expressivo de respostas 'Não Sabe ou Não Respondeu' (NS/NR) em várias questões do instrumento de coleta, em especial nas questões 
referentes aos primeiros ciclos do programa. 2 . $\mathrm{O}$ fato de uma parte dos respondentes não ter estado na condição de gestão que ocupava no momento em que os ciclos anteriores estavam sendo executados, podendo reduzir a precisão das informações sobre os ciclos anteriores. 3. O caráter mais exploratório deste artigo é uma limitação devida, em parte, à inexistência de estudos em profundidade sobre o uso dos recursos do PMAQ-AB em nível municipal, lacuna esta que tentamos ajudar a preencher com o presente artigo, mas que demanda outras pesquisas a serem desenvolvidas. Ainda assim, como o objetivo era identificar a maneira como os gestores atuais valoraram o processo do PMAQ-AB como um todo, considera-se que os dados, ainda que com eventuais imprecisões, são adequados para as análises propostas nesse estudo.

\section{Conclusões}

Conclui-se que os municípios da Paraíba tendiam a utilizar uma forma de gestão homogênea para repasse dos recursos advindos do programa para as EAB participantes do PMAQ-AB. Esse perfil homogêneo se dá pela semelhança nas medidas adotadas pelos municípios, tais quais a adoção do uso da avaliação externa como critério de repasse; a atribuição de um valor percentual sobre a remuneração mensal de cada profissional; o repasse de recursos para outros profissionais, além da equipe mínima; o repasse de $40 \%$ a $50 \%$ dos valores recebidos aos trabalhadores em uma periodicidade semestral ou anual; e a utilização do restante dos recursos em melhorias nas condições de trabalho, em reparos nas unidades, no processo de educação permanente e na compra de medicamentos, insumos e materiais.

Foi possível perceber que os objetivos propostos pelo programa foram alcançados no estado, tendo o resultado da ótica dos gestores como base. Nesse sentido, percebeu-se que o PMAQ-AB, ao transferir recursos financeiros, pontua objetivos para a indução e a ampliação do acesso e da qualidade da atenção, da formação dos profissionais e da melhoria das condições de trabalho. Além disso, o programa se caracterizou como um importante sistema de $\mathrm{P} 4 \mathrm{P}$ para os trabalhadores e induziu o reconhecimento e a valorização das $\mathrm{EAB}$ e dos gestores municipais de saúde na qualificação da $\mathrm{AB}$.

Esta pesquisa permitiu identificar aspectos relevantes sobre como se deu a execução em nível local dos recursos do PMAQ por parte dos municípios de um estado com elevada cobertura de ESF e ampla adesão ao programa. Isso permite identificar potenciais vetores a serem considerados pelos gestores das três esferas, num momento em que esta modalidade de composição do financiamento da $\mathrm{AB}$ foi descontinuada, por meio do Previne Brasil. Por ter sido a primeira experiência em âmbito nacional de repasse de recursos aos municípios vinculados ao alcance de resultados com base em diferentes maneiras de avaliação, compreender o modo como PMAQ foi executado pode ajudar a construir diferentes cenários para superar desafios postos pela maneira em curso de se realizar o financiamento federal para a $\mathrm{AB}$.

\section{Colaboradores}

Rodrigues AWD (0000-0002-3029-0913)* contribuiu substancialmente para concepção e planejamento; análise e interpretação dos dados; elaboração do rascunho; revisão crítica do conteúdo e participou da aprovação da versão final do manuscrito. Mello ECA (0000-0001-9747-2992)* contribuiu substancialmente para concepção e planejamento; análise e interpretação dos dados; elaboração do rascunho e revisão crítica do conteúdo. Candeia RMS (0000-0002-2641-1620)* contribuiu substancialmente para concepção, delineamento do estudo e aquisição; e para análise e interpretação dos dados do trabalho. Silva G (0000-0002-4320-632x)* contribuiu substancialmente para concepção e planejamento; para a análise e interpretação dos dados; contribuiu significativamente para elaboração do rascunho e revisão crítica do conteúdo. Gomes LB (0000-0002-1957-0842)* 
contribuiu substancialmente para concepção e planejamento; para a análise e interpretação dos dados; contribuiu significativamente para elaboração do rascunho; revisão crítica do conteúdo e participou da aprovação da versão final do manuscrito. Sampaio J (0000-0003-0439-5057)*

\section{Referências}

1. Flôres GMS, Weigelt LD, Rezende MS, et al. Gestão pública no SUS: considerações acerca do PMAQ-AB. Saúde debate. 2018; 42(116): 237-247.

2. Pinto HA, Sousa ANA, Ferla AA. O Programa Nacional de Melhoria do Acesso e da Qualidade da Atenção Básica: várias faces de uma política inovadora. Saúde debate. 2014; 38(esp):358-372.

3. Brasil. Ministério da Saúde, Secretaria de Atenção à Saúde, Departamento de Atenção Básica. Programa Nacional de Melhoria do Acesso e da Qualidade da Atenção Básica (PMAQ): manual instrutivo. Brasília, DF: MS; 2011.

4. Brasil. Ministério da Saúde, Secretaria de Atenção à Saúde, Departamento de Atenção Básica. Programa Nacional de Melhoria do Acesso e da Qualidade da Atenção Básica (PMAQ): manual instrutivo. Brasília, DF: MS; 2017.

5. Santos TC, Ozorio JC, Ferreira AV, et al. PMAQ-AB e os pactos do SUS: função dispositivo ou repetição? In: Gomes LB, Barbosa MG, Ferla AA, organizadores. Atenção básica: olhares a partir do programa nacional de melhoria do acesso e da qualidade - (PMAQ-AB). Porto Alegre: Rede Unida; 2016. p. 75-99.

6. Amstrong M. Armstrong's handbook of human resource management practice. 13. ed. London: Kogan Page; 2014. contribuiu substancialmente para concepção e planejamento; para a análise e interpretação dos dados; contribuiu significativamente para elaboração do rascunho; revisão crítica do conteúdo e participou da aprovação da versão final do manuscrito.
7. Poli NP, Faoro NT, Prado JJC, et al. Remuneração variável na Atenção Primária à Saúde: relato das experiências de Curitiba e Rio de Janeiro, no Brasil, e de Lisboa, em Portugal. Ciênc. Saúde Colet. 2016; 21(5):1377-1388.

8. Fontenele MFM. Gestão do desempenho humano: um estudo de caso em um Hospital Geral de Fortaleza (CE). Ciênc. Saúde Colet. 2010; 15(supl1):13151324.

9. Brasil. Ministério da Saúde. Agência Saúde: tire suas dúvidas. Brasília, DF: MS; 2019.

10. Harzheim E. "Previne Brasil": bases da reforma da atenção primária à saúde. Ciênc. Saúde Colet. 2020; 25(4):1189-1196.

11. Neves RG, Flores TR, Duro SMSD, et al. Tendência temporal da cobertura de estratégia de saúde da família no Brasil, regiões e unidades da federação, 20062016. Epid. Serv. saúde. 2018; 27(3):1-8.

12. Huotx R. Métodos quantitativos para as ciências humanas. Lisboa: Instituto Piaget; 2002.

13. Wright BE. Public service and motivation: does mission matter? Public Adm. Review. 2007; 67(1):54-63.

14. Gerhart B. Compensation. In: Wilkinson A, Bacon 
N, Redman T, et al., editores. The SAGE Handbook of Human Resource Management. London: SAGE; 2009.

15. Jales ED, Jales RD, Gomes JGN. O PMAQ-AB enquanto ferramenta de gestão do trabalho em saúde a partir das percepções dos gestores e profissionais de saúde. RSC online. 2017; 6(3):77-97.

16. Armond LP. Motivação pela remuneração por desempenho, justiça organizacional e desempenho humano no trabalho. 2017. [dissertação]. Brasília, DF: Faculdade de Economia, Administração, Contabilidade e Ciência da Informação, Universidade de Brasília; 2017. 120 p.

17. Bertusso FR, Rizzotto MLF. PMAQ na visão de trabalhadores que participaram do programa em Região de Saúde do Paraná. Saúde debate. 2018; 42(117):408419 .

18. Barreto JOM. Pagamento por desempenho em sistemas e serviços de saúde: uma revisão das melhores evidências disponíveis. Ciênc. Saúde Colet. 2015; 20(5):1497-1514.

19. Zermiani TC, Helena BP, Gomes DR. Pagamento por desempenho na atenção primária à saúde em Curitiba-PR: incentivo ao desenvolvimento da qualidade. Soc. Cultura. 2018; 21(2).

20. Sampaio J, Moraes MN, Marcolino EC, et al. PMAQ-AB: a experiência local para a qualificação do programa nacional. Rev enferm UFPE online. 2016; 10(5):4318-4328.

21. Universidade Federal de Minas Gerais. Relatório final diálogos online: remuneração por desempenho na Atenção Básica - $1^{\mathrm{a}}$ e $2^{\mathrm{a}}$ Edição. Belo Horizonte: UFMG; 2014.

22. Neves TCCL, Montenegro LAA, Bittencourt SDA. Produção e registro de informações em saúde no Brasil: panorama descritivo através do PMAQ-AB. Saúde debate. 2014; 38(103):756-770.
23. Faria HP, Werneck M, Santos MA, et al. O processo de trabalho em atenção básica à saúde. 2009. [acesso em 2020 jun 30]. Disponível em: https://www.nescon.medicina.ufmg.br/biblioteca.

24. Assunção AA. Condições de trabalho e saúde dos trabalhadores da saúde. In: Gomez CM, Machado JMH, Pena PGL, organizadores. Saúde do trabalhador na sociedade brasileira contemporânea. Rio de Janeiro: Fiocruz; 2011. p. 453-473.

25. Frigoto V. Gestão do trabalho em saúde: uma análise a partir do PMAQ-AB na macrorregião oeste do Paraná. 2018. [dissertação]. Toledo: Universidade Estadual do Oeste do Paraná; 2018. 100 p.

26. Mendes A, Louvison M. O debate da regionalização em tempos de turbulência do SUS. Saúde e Soc. 2015; 24(2):393-402.

27. Dwek E, Oliveira ALM, Rossi P, organizadores. Austeridade e Retrocesso: impactos sociais da política fiscal no Brasil. 2018. [acesso em 2020 jun 17]. Disponível em: http://brasildebate.com.br/wp-content/ uploads/DOC-AUSTERIDADE_doc3-_L9.pdf.

28. Vianna DM, Nogueira CA, Araujo RS, et al. A educação permanente em saúde na perspectiva do enfermeiro na estratégia de saúde da família. Rev. enferm Cent.-Oeste Min. 2015; 5(2):1658-1668.

29. Castro BMC. Avaliação da atenção básica com foco no processo de trabalho das equipes de saúde através do Programa de Melhoria do Acesso e da Qualidade da Atenção Básica (PMAQ-AB-CICLO II). 2017. [dissertação]. Manaus: Fundação Oswaldo Cruz; Instituto Leônidas e Maria Deane; 2017. 132 p.

30. Moraes PN, Iguti AM. Avaliação do desempenho do trabalhador como forma peculiar de prescrição do trabalho: uma análise do PMAQ-AB. Saúde debate. 2013; 37(98):416-426.

31. Reis ALG. O Programa Nacional para Melhoria do Acesso e da Qualidade na Atenção Básica (PMAQ$\mathrm{AB})$ como indutor de novas práticas em unidades de 
saúde da família no município de Camaçari-BA: relato de experiência. [monografia]. Camaçari: Fundação Oswaldo Cruz; 2018. 50 p.

32. Machado GAB. Organização do processo de trabalho vivenciado pelas equipes de saúde da família do município de São Sebastião do Paraíso/MG, a partir da adesão ao Programa de Melhoria do Acesso e da Qualidade da Atenção Básica (PMAQ-AB). [monografia]. Formiga: Universidade Federal de Minas Gerais; 2018. 45 p.

33. Pinto HA. Múltiplos olhares sobre e a partir do Programa Nacional de Melhoria do Acesso e Qualidade. [dissertação]. [Porto Alegre]: Escola de Enfermagem, Universidade Federal do Rio Grande do Sul; 2014. 231 p.
34. Ditterich RG, Moysés ST, Moysés SJ. O uso de contratos de gestão e incentivos profissionais no setor público de saúde. Cad. Saúde Pública. 2012; 28(4):615625.

35. Feitosa RMM, Paulino AA, Lima JJOS, et al. Mudanças ofertadas pelo Programa Nacional de Melhoria do Acesso e da Qualidade da Atenção Básica. Saúde soc. $2016 ; 25(3): 821-829$.

36. Sossai TA, Galavote HS, Vieira ECL, et al. Evidências sobre o Programa Nacional de Melhoria do Acesso e da Qualidade da Atenção Básica. Revista Brasileira de Pesquisa em Saúde. 2016; 18(1):111-119.

Recebido em 07/12/2020

Aprovado em 10/09/2021

Conflito de interesses: inexistente

Suporte financeiro: não houve 